\title{
Exclusividade na coleta de material para exame de colpocitologia oncótica: percepção dos enfermeiros
}

Exclusivity in the collection of material for oncotic colpocytology examination: nurses' perception

Exclusividad en la recolección de material para el examen de colpocitología oncótica: percepción de los enfermeros

\section{Letícia Lima de Oliveira ${ }^{\mathrm{I}}$, Marllon Rodrigo Sousa Santos ${ }^{\mathrm{II}}$, Ivaneide Leal Ataíde Rodrigues ${ }^{\mathrm{III}}$

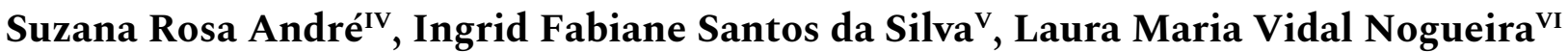

\begin{abstract}
Resumo: Objetivo: analisar a percepção dos enfermeiros a respeito da exclusividade da coleta de material para a realização de colpocitologia oncótica. Método: estudo descritivo de natureza qualitativa, tendo como participantes 18 enfermeiros de Unidades Municipais de Saúde de Belém-Pará. A coleta de dados ocorreu entre junho e agosto de 2017 com auxílio de um roteiro de entrevista semiestruturado, e utilizou-se análise de conteúdo para interpretação dos resultados. Resultados: os dados correspondem a percepções sobre a coleta do exame de colpocitologia oncótica, subdivididas em percepções sobre a coleta realizada pelo enfermeiro e técnico de enfermagem, facilidades e dificuldades na coleta e alterações na rotina dos enfermeiros. Considerações finais: as percepções dos enfermeiros são heterogêneas, porém convergem ao reconhecerem a necessidade de agregar conhecimento técnico e científico para realização da coleta de material para colpocitologia oncótica, em razão da alta complexidade da técnica.
\end{abstract}

Descritores: Teste de papanicolau; Enfermagem; Legislação de enfermagem; Neoplasias do colo do útero

Abstract: Aim: To analyze nurses' perceptions about the exclusivity of material collection for oncotic
colpocytology. Method: a descriptive study of qualitative nature, having as participants 18 nurses from Municipal

I Enfermeira. Mestranda em enfermagem. Universidade do Estado do Pará, Belém, Pará, Brasil, letlima06@gmail.com, https://orcid.org/00000003-3984-625X

II Enfermeiro. Residente em oncologia. Universidade Federal do Pará, Belém, Pará, Brasil, marlon-rodrigo@hotmail.com, https://orcid.org/00000002-7816-924X

III Enfermeira. Doutora em Enfermagem. Universidade do Estado do Pará, Belém, Pará, Brasil, ilar@globo.com, https://orcid.org/0000-00019968-9546

IV Enfermeira. Doutoranda em enfermagem. Universidade Federal do Rio de Janeiro, Rio de Janeiro, Rio de Janeiro, Brasil, suzanarandre@gmail.com, https://orcid.org/0000-0002-4219-9279

V Enfermeira. Doutoranda em enfermagem. Universidade Federal do Rio de Janeiro, Rio de Janeiro, Rio de Janeiro, Brasil, ingridenfermeir@gmail.com, https://orcid.org/0000-0002-9191-833X

VI Enfermeira. Doutora em Enfermagem. Universidade do Estado do Pará, Belém, Pará, Brasil, lauramavidal@gmail.com, https://orcid.org/00000003-0065-4509 
Health Units of Belém-Pará. Data collection took place between June and August 2017 with the aid of a semistructured interview script, and content analysis was used to interpret the results. Results: the data correspond to perceptions about the collection of the oncotic colpocytology exam, subdivided into perceptions about the collection performed by the nurse and nursing technician, facilities and difficulties in collecting and changes in the routine of nurses. Conclusion: the perceptions are heterogeneous, but converge in recognizing the need for nurses to add technical and scientific knowledge to perform the collection of material for oncotic colpocytology, due to the high complexity of the technique.

Descriptors: Papanicolaou Test; Nursing; Legislation, Nursing; Uterine Cervical Neoplasms

Resumen: Objetivo: analizar las percepciones de los enfermeros sobre la exclusividad de la recolección de material para la colpocitología oncótica. Método: estudio descriptivo de naturaleza cualitativa, teniendo como participantes 18 enfermeros de las Unidades Municipales de Salud de Belém-Pará. La recopilación de datos se realizó entre junio y agosto de 2017 con la ayuda de un guión de entrevista semiestructurada, y se utilizó el análisis de contenido para la interpretación de los resultados. Resultados: los datos corresponden a percepciones sobre la recolección del examen de colpocitología oncótica, subdividido en percepciones sobre la recolección realizada por el enfermero y el técnico de enfermería, instalaciones y dificultades en la recolección y cambios en la rutina de los enfermeros. Conclusión: las percepciones son heterogéneas, pero convergen en el reconocimiento de la necesidad de que los enfermeros agreguen conocimientos técnicos y científicos para la realización de la recolección de material para la colpocitología oncótica, debido a la alta complejidad de la técnica.

Descriptores: Prueba de Papanicolaou; Enfermería; Legislación de Enfermería; Neoplasias del Cuello Uterino

\section{Introdução}

A prevenção e o controle do câncer de colo de útero têm constituído grandes desafios aos serviços e aos profissionais figurando com prioridade nas agendas de saúde, demandando ações estratégicas em todo o Brasil, sendo considerado um problema de saúde pública. Segundo estimativas, no Brasil, para o biênio de 2018-2019, calcula-se 16.370 novos casos de câncer do colo do útero no Brasil, com risco estimado de 15,43/100 mil mulheres. ${ }^{1}$

A Região Norte ocupa o primeiro lugar em incidência, com número estimado para o mesmo ano de 2.300 casos novos. O Estado do Pará está na quarta posição em taxa bruta de adoecimento com 860 casos novos, sendo a incidência estimada para 2018 de 20,55/100 mil habitantes, com expressiva concentração na capital Belém (280 casos - 33,69/100 mil hab.), sendo a segunda neoplasia que mais acomete mulheres no Estado do Pará, precedida apenas pelo câncer de mama (sem considerar os tumores de pele não melanoma). ${ }^{1}$ 
Por ser uma doença de evolução lenta até se tornar invasivo, o câncer de colo uterino e suas lesões precursoras são passíveis de rastreamento, detecção precoce e tratamento quando diagnosticados em suas fases iniciais. A principal estratégia utilizada para o rastreamento da doença é por meio do exame de colpocitologia oncótica ou Papanicolau, devido a sua sensibilidade, especificidade, baixo custo, segurança na execução e aceitação pelas mulheres, sendo recomendado no Brasil para as mulheres sexualmente ativas e com idade entre 25 aos 64 anos de idade. ${ }^{2-3}$

De acordo com a Resolução do Conselho Federal de Enfermagem (COFEN) n 385/2011, a coleta de material para exame de colpocitologia oncótica, que antes podia ser executada por enfermeiros, técnicos de enfermagem e auxiliares de enfermagem, deve ser realizada exclusivamente por enfermeiros por considerar a magnitude epidemiológica, econômica e social do câncer do colo do útero, por ser um procedimento complexo com demanda técnica e científica que requer processo de trabalho organizado e sistematizado da assistência de enfermagem. ${ }^{4}$

Entretanto, há controvérsias na aplicação dessa Resolução, tendo em vista que abrange todo o território nacional e não leva em consideração a diversidade na epidemiologia da doença, a estrutura dos serviços de saúde e as particularidades de cada região, o que levou o Instituto Nacional do Câncer (INCA) a alertar para possíveis consequências na aplicação desse instrumento legal, ponderando que a coleta realizada não apenas por enfermeiros, mas também por auxiliares e técnicos de enfermagem, desde que bem treinados, são estratégias para a ampliação da cobertura do exame..$^{5}$

Ainda nesse contexto, a Secretaria de Estado de Saúde Pública do Pará (SESPA) emitiu Nota Técnica contrariando a Resolução COFEN n 385/2011 autorizando a realização da coleta por técnicos e auxiliares de enfermagem no intuito de garantir o acesso das mulheres ao exame, tendo em vista que no Estado a maior parte das coletas era realizada por técnicos de 
enfermagem. Em relação a esta Nota, o Conselho Regional de Enfermagem (COREN-PA) reagiu afirmando que a SESPA tentava transferir para a enfermagem um problema de gestão em vez de buscar novas estratégias para garantir as políticas de saúde. Esta discussão foi levada ao Conselho Estadual de Saúde que ratificou o posicionamento da SESPA, recomendando a cooperação entre diversas instituições para a reavaliação da Resolução. No entanto, a determinação legal deliberada pelo COFEN aos profissionais de enfermagem permanece em vigência ${ }^{6}$

A partir desse cenário, questionou-se: Qual a percepção dos enfermeiros a respeito da exclusividade na coleta de material para exame de colpocitologia oncótica após determinação da Resolução COFEN no 385/2011? E definiu-se como objetivo: analisar a percepção dos enfermeiros a respeito da exclusividade da coleta de material para a realização de colpocitologia oncótica.

\section{Método}

Trata-se de um estudo descritivo de natureza qualitativa, realizado em 18 Unidades Municipais de Saúde (UMS) gerenciadas pela Secretaria Municipal de Saúde (SESMA) do município de Belém, no Estado do Pará, que representa 85,7\% do total de UMS da cidade. Sobre os participantes, foram selecionados conforme disponibilidade no momento da coleta, um enfermeiro de cada UMS, totalizando 18 , sendo critério de inclusão estar realizando a coleta de material para exame de colpocitologia oncótica, independentemente do tempo de atuação neste serviço.

Para obtenção dos dados utilizou-se um roteiro de entrevista semiestruturado elaborado pelos pesquisadores, composto por dois eixos: dados sociodemográficos que incluíam a faixa etária, sexo, formação profissional, tempo de serviço e de atuação na coleta da colpocitologia; e questões específicas relacionadas ao objeto estudado, contemplando o conhecimento a respeito 
da Resolução 385/2011 - COFEN e a opinião pessoal sobre a exclusividade da coleta pelo enfermeiro; implicações que reconhecem na execução da atividade, assim como o nível de preparo e satisfação.

A interpretação dos resultados baseou-se na análise de conteúdo, observando os passos: leitura geral do material; codificação para categorização do material; recorte do material em unidades de registro com semelhança; agrupamento das unidades de registro em categorias comuns; agrupamento progressivo das categorias de forma técnica e sistemática até a categorização final; inferência e interpretação dos resultados com base no referencial teórico do estudo. ${ }^{7}$

O estudo foi aprovado no Comitê de Ética em Pesquisa do Curso de Graduação em Enfermagem da Universidade do Estado do Pará em 26 de maio de 2017, sob o parecer de nº 2.084.826.

\section{Resultados}

Os dados correspondem a caracterização sociodemográfica dos participantes e dizem respeito a faixa etária, sexo, formação profissional, tempo de serviço e de atuação na coleta da colpocitologia. Os resultados referentes ao objeto de estudo encontram-se organizados em uma categoria: percepções sobre a coleta do exame de colpocitologia oncótica que se subdivide em quatro subcategorias que abordam a coleta de material realizada pelo enfermeiro, a coleta realizada pelo técnico de enfermagem, as facilidades e dificuldades na realização da coleta do material e as alterações na rotina dos enfermeiros que expressam suas diferentes percepções frente à exclusividade na coleta da colpocitologia oncótica.

Em relação a idade dos participantes, 7 (38;89\%) estavam na faixa etária dos 31 a 40 anos, seguidos dos intervalos de 41 a 50 anos e 51 a 60 anos com 5 (27;78\%) participantes cada, e 1 $(5 ; 56 \%)$ entre os 18 a 30 anos. Quanto ao sexo, predominou o feminino com $16(88 ; 89 \%)$ participantes. No que se refere à formação profissional, $13(72 ; 22 \%)$ possuíam pós-graduação. 
Considerando o tempo em que atuavam na atenção básica, 7 (38;89\%) desenvolviam atividades entre 1 e 5 anos, seguido dos intervalos de 6 a 10 anos com 4 (22;22\%) enfermeiras (os), e mais de 10 anos com 7 (38;89\%) participantes. Quanto ao tempo de atuação na coleta de material para realização do exame, $4(22 ; 22 \%)$ coletavam há menos de 1 ano, 10 (55;56\%) enfermeiros atuavam entre 1 e 5 anos, e $4(22 ; 22 \%)$ coletavam há mais de 5 anos.

Quando perguntados sobre o conhecimento a respeito da resolução COFEN n 385/2011 e como tiveram acesso à mesma, $17(94 ; 44 \%)$ referiram variadas fontes de acesso à informação, 9 (52;94\%) foram comunicados pela instituição em que trabalham, $3(17 ; 65 \%)$ por meio do COFEN/COREN e 5 (29;41\%) tiveram acesso pelas mídias sociais.

A respeito da oferta de capacitação no serviço, 10 (55;56\%) participantes informaram terem sido capacitados mediante oferta da SESPA ou SESMA, e $8(44 ; 44 \%)$ participantes afirmaram não ter tido nenhuma capacitação no serviço, tendo apenas o conhecimento adquirido por ocasião da graduação:

[...] foi só pela faculdade mesmo, o basicão, que é uma só paciente que a gente faz, durante a graduação toda. (E8)

\section{Percepções sobre a coleta do exame de colpocitologia oncótica}

A categoria contempla a visão dos participantes quanto a coleta de material para exame de colpocitologia oncótica realizada pelo enfermeiro, sobre a realização da mesma pelo técnico de enfermagem, as dificuldades e facilidades para sua realização e as alterações na rotina dos enfermeiros.

\section{Percepções sobre a coleta realizada pelo enfermeiro}

Dentre os participantes do estudo, $13(72,22 \%)$ reconheceram a alta complexidade da coleta de material para exame de colpocitologia oncótica. Observou-se que a maioria dos enfermeiros 
que expressavam esse posicionamento demonstravam afinidade com a execução da técnica e desenvolviam apenas esta atividade na unidade de saúde o que aparece, expresso nas falas:

eu acho que o enfermeiro é um profissional habilitado para coletar o PCCU [Preventivo do Câncer do Colo do Útero], uma vez que é um procedimento que exige tomada de decisão e orientação voltada para cada paciente, além de exigir conhecimento a respeito da patologia e das doenças que acometem o aparelho reprodutor feminino. (E11)

eu acho muito bom que seja feito por pessoas mais capacitadas, com mais escolaridade, com mais discernimento [...] acho importante. (E3)

Não obstante, os enfermeiros reconheceram que a exclusividade da coleta ocasionou sobrecarga de trabalho, tendo em vista que as UMS mantiveram o mesmo quantitativo de profissionais com as mesmas atribuições, sem ampliar o quadro de enfermeiros, conforme depoimentos:

é um pouco complicado porque a gente não pode parar de atender os programas para ficar exclusivo só para coleta do preventivo. Tem muito programa para atender e tem poucas pessoas para fazer o atendimento. (E1)

eu acho que nós já temos muito trabalho, a gente já tem muita coisa para fazer [...] Da assistência ao setor administrativo já é todo nosso, entendeu? Eu acho que foi mais um serviço para nós. Tem alguns colegas nossos que acham que foi bom que a gente ganha espaço e tudo, mas eu não vejo por aí. (E4)

\section{Percepções sobre a coleta realizada pelo técnico de enfermagem}

Segundo a opinião de $5(27,78 \%)$ enfermeiros, a coleta do exame poderá ser realizada pelo técnico de enfermagem, desde que este seja bem treinado e supervisionado pelo enfermeiro. Trata-se de um discurso que demonstra credibilidade na atuação do técnico para realização do 
procedimento. Observou-se que os enfermeiros que expressaram esse posicionamento eram responsáveis por vários programas de assistência à saúde nas UMS:

o técnico ajudaria muito, um técnico treinado. (E1)

a coleta em si é só técnica e como tal eu posso ensinar para qualquer pessoa. A técnica em si eu não vejo como 'é preciso ser exclusivo do enfermeiro', porque eu acho que a técnica bem aprendida pelo próprio técnico de enfermagem, pode ser realizada [...]. (E17)

Evidenciou-se ainda que $3(16,67 \%)$ participantes teceram críticas à realização da coleta pelos técnicos por acreditarem que não apresentam a mesma qualidade daquela realizada pelo enfermeiro. Ainda, fortaleceram seus discursos referindo a falta de habilidade por ocasião da recepção da paciente, sem o acolhimento necessário comprometendo, inclusive, o retorno para acesso ao resultado do exame e o seguimento terapêutico, ilustrado nas falas:

[...] deite aqui, bote seu pé aqui [...] Não tem aquele cuidado de pedir para paciente relaxar, de conversar com ela, de realmente olhar qual é a situação dela [...] As técnicas falam demais, elas dizem que o colo está sangrando, que aquilo está vermelho, fazem assim um terror para paciente, que elas não vêm nem buscar o resultado, entendeu? Acabam dando informações desnecessárias. (E5)

Percepções sobre as dificuldades e facilidades para a coleta do exame de colpocitologia oncótica

Dentre as dificuldades relatadas pelos enfermeiros com implicações diretas na realização do exame, destacou-se a falta de recursos materiais; o espaço físico inadequado ou compartilhado; a sobrecarga de trabalho e a coleta realizada por profissionais do sexo masculino. Tais situações estão ilustradas nas falas seguintes:

a minha dificuldade aqui é com materiais. Teve um período aqui, por exemplo, não tinha o tubete. (E17)

nós não temos uma sala só para a coleta do PCCU, esta sala aqui tem médico que atende. (E16) 
por eu ser homem é um muro muito grande. Eu tenho que dar palestra, conversar com todas e só as idosas que já aceitam, as outras não aceitam. (E9)

Para os enfermeiros, as facilidades identificadas na rotina das UMS dizem respeito ao atendimento por demanda espontânea que favorece a procura pelo exame e facilita o acesso; ao apoio da gestão; ser enfermeiro exclusivo para coleta do exame, ilustradas nos depoimentos:

a facilidade é que a gente atende todos os dias e é porta aberta, basta chegar. (E2)

apoio da gestão, da gerência da unidade, para gente executar esse trabalho. Isso aí é uma facilidade muito grande, entendeu? Tem os equipamentos mínimos necessários [...]. (E5)

a unidade daqui, em relação a algumas outras, tem um perfil diferente, a gente tem uma quantidade de enfermeiros bem maior, então eu consigo ser exclusiva do PCCU[...]. (E17)

\section{Percepções sobre as alterações na rotina dos enfermeiros}

Contempla discursos que apontam as implicações na rotina dos enfermeiros após a vigência da resolução COFEN n 385/2011 estando, na maioria das vezes, relacionadas ao déficit de recursos humanos e a organização do processo de trabalho, ocasionando sobrecarga e acúmulo de funções.

O advento da Resolução impulsionou tomada de decisão por alguns enfermeiros em relação ao serviço de coleta, em detrimento da orientação dos gestores das Unidades e da gestão da saúde no município de Belém, objetivando a não interrupção da coleta de material para exame de PCCU, que se sobressaem nos seguintes depoimentos:

é, a gente está pegando o técnico que tem o curso de enfermagem [Técnicos de Enfermagem que cursaram graduação em enfermagem], para não ter tanto problema assim para gente, nem para eles. Se eu tivesse que 
Exclusivity in the collection of material for oncotic colpocytology examination... $\mid 10$

realmente ficar fazendo coleta, ia atrapalhar o atendimento dos pacientes, ia ter que parar o atendimento dos programas. (E1)

um dia que eu deixo de atender gestantes, crianças, tuberculose, hanseníase [...] aí nos outros dias a gente já tem que agendar mais gente. (E3)

a gente teve que fazer uma reunião e dizer assim: nesse dia a gente não agenda o pré-natal, a gente tem que fazer malabarismo para poder iniciar o pré-natal na data preconizada, então isso aí já altera a minha rotina sim! [...] Eles colocaram essa resolução contando que iriam contratar mais enfermeiros e não contrataram, entendeu?(E16)

eu fui, justamente após a resolução, para o PCCU, porque anteriormente era um técnico de enfermagem. Então, eu acho que mudou muito mais na rotina da unidade de se adequar a resolução do que na minha. Eu era da urgência, vim para o ambulatório com essa intenção, de vir para o PCCU [...]. (E17)

\section{Discussão}

O perfil sociodemográfico dos participantes evidenciou prevalência do sexo feminino, por ser a enfermagem uma profissão marcadamente feminina desde sua origem, e por ser este um procedimento relacionado à intimidade da mulher. Neste caso, o sexo se torna elemento relevante para o desempenho da função, podendo contribuir ou comprometer o rastreamento e prevenção do câncer do colo do útero. ${ }^{8-9}$ Trata-se de um aspecto a ser valorizado, sobretudo em unidades em que há somente um enfermeiro, não permitindo escolha pelas mulheres.

Neste estudo, a maioria dos participantes atuava na coleta de material para colpocitologia oncótica por até 5 anos, coincidindo com o período em que a Resolução COFEN n $385 / 2011$ entrou em vigor, evidenciando que a decisão do órgão regulador foi a motivação para alocar os enfermeiros na sala de coleta. E apenas 55,56\% (10) dos entrevistados passaram por capacitação, 
11 | Oliveira LL, Santos MRS, Rodrigues ILA, André SR, Silva IFS, Nogueira LMV

e parte destes afirmaram que foi pela necessidade de atender a resolução do COFEN, ocasionando implicações não só para os enfermeiros, mas também para a gestão do serviço.

No entanto, embora este percentual seja relativamente baixo, um estudo realizado com enfermeiros no Estado da Bahia apontou que a busca por qualificação vem aumentando entre os profissionais enfermeiros, principalmente, no que diz respeito a programas de pós-graduação. ${ }^{10}$ A educação permanente deve ocorrer de forma sistemática na atenção básica para qualificar as práticas em saúde, e todos os profissionais que realizam a coleta de material para exame de colpocitologia oncótica devem receber capacitação. ${ }^{5}$

Embora a mudança na rotina dos enfermeiros seja percebida de forma distinta apontando percepções positivas e negativas para o serviço, os discursos dos participantes mostram coerência com as justificativas constantes na Resolução 385/2011 - COFEN no que se refere a complexidade do exame, demandando não apenas conhecimento técnico como científico e citação das atribuições do enfermeiro dispostas na Lei do exercício da profissão nº 7.498/1986. ${ }^{9}$

Nesse sentido, a atuação do enfermeiro é considerada essencial na prevenção do câncer do colo do útero, visto que a atenção básica abrange desde o acolhimento e sensibilização das mulheres até o diagnóstico, por meio da realização da coleta de material para exame de colpocitologia oncótica. Desta forma, entende-se que há necessidade de o enfermeiro ser capacitado para atuar nas diversas atividades para o controle da doença. ${ }^{11}$

Ainda nessa perspectiva, um estudo relacionado à importância da assistência de enfermagem na prevenção do câncer de colo uterino, evidenciou o olhar diferenciado das usuárias assistidas pelo enfermeiro com aprovação de sua abordagem e orientação adequada às suas demandas. Além disso, reconhecem que a atuação dos mesmos é essencial para a redução de novos casos da doença. ${ }^{12}$

A decisão de incorporar a coleta de material para colpocitologia oncótica como exclusiva do enfermeiro alterou a rotina e o processo de trabalho nas UMS ocasionando, na percepção dos 
profissionais, acúmulo de tarefas e necessidade de reorganização dos serviços. Este cenário se configurou para os participantes como uma grande dificuldade decorrente da Resolução $\mathrm{n}^{\circ}$ $385 / 2011$.

Em suas falas, os participantes reconheceram que a implementação da resolução implicou na sobrecarga de trabalho, tendo em vista que não foram consideradas as inúmeras atribuições e atividades que o enfermeiro desempenha dentro do contexto da atenção básica, direcionadas para os indivíduos e suas coletividades. Sejam estas: educação em saúde voltada para a prevenção e promoção da saúde, assistência e gerenciamento dos serviços de saúde em todos os ciclos da vida e, ainda, diagnóstico, tratamento e recuperação dos agravos de saúde. ${ }^{13-15}$

A demanda excessiva de trabalho correlaciona-se com as falas dos enfermeiros que admitiram delegar a coleta para o técnico de enfermagem, a fim de viabilizar o desenvolvimento de todas as suas atribuições nas UMS, embora reconheçam que para tal, estes precisam de capacitação e supervisão direta, essencialmente para o acolhimento às mulheres. Isto ocorre porque embora a enfermagem seja imprescindível para o acesso e funcionamento dos serviços de saúde, ainda persistem desequilíbrios e lacunas na disponibilidade, distribuição, composição, qualificação e produtividade desses profissionais. ${ }^{16}$

A percepção de sobrecarga de trabalho como implicação mais impactante, se deu pelo número insuficiente de enfermeiros nos serviços, fazendo-os atribuir a esse fato o possível comprometimento da cobertura e qualidade do serviço de coleta do material. Desse modo, as instituições/organizações de saúde devem promover condições de trabalho adequadas, número de profissionais e remuneração compatíveis com o serviço, reduzindo desta forma o risco de danos ao paciente. Vale ressaltar que grande parte dos profissionais enfermeiros possui vários empregos e que há alta rotatividade nos serviços devido à baixa remuneração na profissão e ainda condições de trabalho inadequadas que os expõem a níveis alarmantes de estresse ${ }^{17}$, o que também foi identificado durante as entrevistas. 
Pode-se afirmar também que os enfermeiros não são os únicos profissionais que prestam assistência ao paciente. No entanto, assumem posição de liderança diante da equipe multiprofissional e da comunidade. São responsáveis pelo treinamento e capacitação de outras categorias de enfermagem, além de avaliar, sistematizar e decidir a conduta mais apropriada para cada indivíduo, colocando em prática habilidades de gestão e gerenciamento de recursos, profissionais e informações em saúde ${ }^{17-18}$, ocasionando o acúmulo de funções identificado na rotina das UMS.

\section{Considerações finais}

As percepções dos enfermeiros a respeito da exclusividade na coleta de colpocitologia oncótica, imposta pela Resolução COFEN n 385/2011, não são homogêneas, no entanto, embora exista divergência de opinião, observa-se convergência ao reconhecerem a necessidade de o enfermeiro agregar conhecimento técnico e científico para execução da atividade, dada sua alta complexidade. Reconhecem ainda que a implementação da resolução do COFEN ocasiona sobrecarga de trabalho para a categoria.

A coleta exclusiva por enfermeiros foi efetivada como adição de tarefas, em meio a (des)estruturação do serviço público de saúde local, mediada pela fiscalização do órgão de classe, ocasionando implicações na rotina das unidades de saúde. Tais implicações não são consequências de uma atitude equivocada e sem fundamentos do conselho de classe que rege a profissão, mas sim do despreparo do município para a readequação das unidades frente a mudança.

É oportuno destacar a importância desta resolução, tendo em vista que a sua efetivação poderá contribuir para o atendimento mais qualificado às usuárias, e também para melhor demarcação do espaço profissional dos enfermeiros. Entretanto, ressente-se de melhor estruturação do serviço de coleta para o exame, contemplando as normas ministeriais, 
implementação de programas de capacitação permanente para os enfermeiros e readequação do quadro de pessoal para assegurar o bom funcionamento de todos os programas e ações na atenção básica. É essencial que haja empoderamento pelos enfermeiros para que exerçam sua profissão com compromisso e qualidade, primando sempre pelos preceitos éticos estabelecidos na lei de exercício profissional, a fim de garantir melhorias na assistência aos usuários.

Com base nesse cenário, sugere-se a ampliação deste estudo para outros municípios e Estados, visto que a vigência da resolução se aplica a todo o território nacional, assim como a realização de pesquisas comparativas nos serviços público e privado, bem como estudos relativos a qualidade do atendimento e coleta de material para o exame de colpocitologia oncótica realizada pelo enfermeiro e pelo técnico de enfermagem, de modo a justificar ou não a implantação da resolução pelo COFEN.

As limitações deste estudo dizem respeito à ausência de comparações com demais setores ou instâncias que realizam a coleta da colposcopia oncótica, limitando-se aos profissionais das Unidades Básicas de Saúde, sem que tenham sido incluídos enfermeiros das Estratégias Saúde da Família, bem como os profissionais que realizam a coleta na rede privada.

\section{Referências}

1. Ministério da Saúde (BR). Estimativa 2018: câncer do colo do útero [Internet]. Rio de Janeiro: Instituto Nacional do Câncer; 2018 [acesso em 2019 nov 17]. Disponível em: http://www1.inca.gov.br/estimativa/2018/sintese-de-resultados-comentarios.asp

2. Brasil. Conselho Federal de Enfermagem. Resolução $n^{\circ} 385$, de 3 de outubro de 2011. Altera o termo inicial de vigência da Resolução Cofen $n^{\circ}$ 381, de 18 de julho de 2011, que normatiza a normatização da execução, pelo Enfermeiro, da coleta de material para colpocitologia oncótica pelo método de Papanicolau [Internet]. 2011 [acesso em 2017 fev 23]. Diário Oficial da União, Brasília (DF); 2011 out 06. Seção $1, \quad$ p. 151 . Disponível em: http://www.cofen.gov.br/wpcontent/uploads/2012/03/resolucao_385_2011.pdf

3. Brasil. Instituto Nacional do Câncer. Ofício n 717 , de 30 de agosto de 2011. Resolução COFEN N ${ }^{\circ} 381$ sobre coleta de material para realização do exame citopatológico por auxiliares e técnicos de enfermagem [Internet]. Brasília (DF): Instituto Nacional do Câncer; 2011 [acesso em 2017 mar 15]. 
Disponível em: https://www.inca.gov.br/sites/ufu.sti.inca.local/files//media/document//oficiono717.2011gab.incade30.08.11.pdf

4. Silva ERP, Souza AS, Souza TGB, Tsuha DH, Barbieri AR. Screening for cervical cancer in imprisoned women in Brazil. PLoS ONE [Internet]. 2017 [acesso em 2018 jul 25];12(12):1-15. Disponível em: http://journals.plos.org/plosone/article/file?id=10.1371/journal.pone.0187873\&type=printable

5. Barbosa IR. Regional and socioeconomic differences in the coverage of the Papanicolau test in Brazil: data from the Brazilian health survey 2013. Rev Bras Med Ginecol Obstet [Internet]. 2017 [acesso em 2018 jul 25]:39(9):480-7. Disponível em: http://www.scielo.br/pdf/rbgo/v39n9/0100-7203-rbgo-39-09-00480.pdf

6. Governo do Estado do Pará, Secretaria de Estado de Saúde Pública, Conselho Estadual de Saúde do Pará (CES/PA). Resolução CES/Pará n 010, de 21 de fevereiro de 2017. Aprova o plano de ação da vigilância sanitária do Estado do Pará, com recomendações [Internet]. 2017 fev 21 [acesso em 2017 nov 12]. Disponível em: https:/www.jusbrasil.com.br/diarios/139442120/doepa-09-03-2017-pg27?ref=previous_button

7. Bardin L. Análise de Conteúdo. São Paulo: Edições 70, 2011.

8. Aguilar RP, Soares DA. Barreiras à realização do exame Papanicolau: perspectivas de usuárias e profissionais da Estratégia de Saúde da Família da cidade de Vitória da Conquista-BA. Physis (Rio J) [Internet]. 2015 [acesso em 2017 fev 21];25(2):359-79. Disponível em: http://dx.doi.org/10.1590/S010373312015000200003

9. Brasil. Presidência da República. Casa Civil. Subchefia para Assuntos Jurídicos. Lei $n^{\circ} 7.498$, de 25 de junho de 1986. Dispõe sobre a regulamentação do exercício da enfermagem, e dá outras providências [Internet]. 1986 jun 25 [acesso em 2017 nov 01]. Disponível em: http://www.planalto.gov.br/ccivil_03/leis/L7498.htm

10. Machado LSF, Rodrigues EP, Oliveira LMM, Laudano RCS, Nascimento Sobrinho CL. Agravos à saúde referidos pelos trabalhadores de enfermagem em um hospital público da Bahia. Rev Bras Enferm [Internet]. 2014 [acesso em 2018 jan 04];67(5):684-91. Disponível em: http://dx.doi.org/10.1590/00347167.2014670503

11. Sebold LF, Suave S, Girondi JBR, Kempfer SS, Echevarría-Guanilo ME. A percepção de mulheres sobre o exame preventivo de câncer uterino e os seus resultados. J Nurs Health [Internet]. 2017 [acesso em 2017 out 20];7(2):164-77. Disponível em: https://periodicos.ufpel.edu.br/ojs2/index.php/enfermagem/article/view/9877/7886

12. Cunha ES. Assistência de enfermagem na prevenção do câncer de colo uterino. FACIDER Rev Científica. 2016;9:1-16.

13. Barbiani R, Dalla Nora CR, Schaefer R. Práticas do enfermeiro no contexto da atenção básica: scoping review. Rev Latinoam Enferm [Internet]. 2016 [acesso em 2018 maio 25];24:e2721. Disponível em: 
Exclusivity in the collection of material for oncotic colpocytology examination... $\mid 16$

http://www.scielo.br/pdf/rlae/v24/pt_0104-1169-rlae-24-02721.pdf

doi:http://dx.doi.org/10.1590/15188345.0880 .2721

14. Moreno CA, Ferraz LR, Rodrigues TS, Lopes AOS. Atribuições dos profissionais de enfermagem na estratégia de saúde da família, uma revisão das normas e práticas. Rev Bras Ciênc Saúde [Internet]. 2015 [acesso em 2018 maio 20];19(3):233-40; Disponível em: http://www.periodicos.ufpb.br/index.php/rbcs/article/view/23355/15075

15. Ferreira SRS, Périco LAD, Dias VRFG. The complexity of the work of nurses in Primary Health Care. Rev Bras Enferm [Internet]. 2018 [acesso em 2018 jun 25];71(Supl 1):704-9. Disponível em: http://www.scielo.br/scielo.php?script=sci_arttext\&pid=S0034-71672018000700704\&lng=pt

16. Pires DEP, Machado RR, Soratto J, Schere MA, Gonçalves ASR, Trindade LL. Cargas de trabalho da enfermagem na saúde da família: implicações no acesso universal. Rev Latinoam Enferm [Internet]. 2016 [acesso em 2018 jul 25];24:2682. Disponível em: http://www.scielo.br/pdf/rlae/v24/pt_0104-1169-rlae-09922682.pdf

17. Novaretti MCZ, Santos EV, Quitério LM, Daud-Gallotti RM. Sobrecarga de trabalho da enfermagem e incidentes e eventos adversos em pacientes internados em UTI. Rev Bras Enferm [Internet]. 2014 [acesso em 2017 nov 01];67(5):692-9. Disponível em: http://www.scielo.br/pdf/reben/v67n5/0034-7167reben-67-05-0692.pdf

18. Vieira MA, Souto LES, Souza SM, Lima CA, Ohara CVS, Domenico EBL. Diretrizes Curriculares Nacionais para a área da enfermagem: o papel das competências na formação do enfermeiro. Renome Rev Norte Mineira Enferm [Internet]. 2016 [acesso em 2018 jan 04];5(1):105-21. Disponível em: http://www.renome.unimontes.br/index.php/renome/article/view/102/148

\section{Autor correspondente}

Suzana Rosa André

E-mail: suzanarandre@gmail.com

Endreço: R. Afonso Cavalcanti, 275 - Cidade Nova, Rio de Janeiro - RJ, CEP: 20211-130

\section{Contribuições de Autoria}

\section{1 - Letícia Lima de Oliveira}

Concepção e planejamento do projeto de pesquisa, obtenção ou análise e interpretação dos dados, redação

\section{2 - Marllon Rodrigo Sousa Santos}

Concepção e planejamento do projeto de pesquisa, obtenção ou análise e interpretação dos dados, redação 
17 | Oliveira LL, Santos MRS, Rodrigues ILA, André SR, Silva IFS, Nogueira LMV

\section{3 - Ivaneide Leal Ataíde Rodrigues}

Interpretação dos dados, revisão crítica

\section{4 - Suzana Rosa André}

Interpretação dos dados, revisão crítica

\section{5 - Ingrid Fabiane Santos da Silva}

Interpretação dos dados, revisão crítica

\section{6 - Laura Maria Vidal Nogueira}

Concepção e planejamento do projeto de pesquisa, redação e revisão crítica

\section{Como citar este artigo}

Oliveira LL, Santos MRS, Nogueira LMV, Rodrigues ILA, André SR, Silva IFS. Exclusividade na coleta de material para exame de colpocitologia oncótica: percepção dos enfermeiros. Rev. Enferm. UFSM. 2020 [Acesso em: Anos Mês Dia];vol.10, e15: 1-17. DOI:https://doi.org/10.5902/2179769233721 